\title{
Magnetic properties of nitroxide multiradicals
}

\author{
André RASSAT \\ Laboratoire de Chimie, Ecole Normale Supérieure, 24 Rue Lhomond \\ F 75231 PARIS Cedex 05
}

\begin{abstract}
After a discussion of previous results on nitroxide mono- and multiradicals, magnetic properties of new bi- and tetraradicals are presented.
\end{abstract}

Magnetic properties of a molecule may refer to the isolated species or to its condensed phase (1). Typical individual properties of monoradicals are $g$ values and hyperfine coupling constants. In multiradicals (reserving polyradicals for polymers obtainedfrom stable radicals as monomer), dipolar $\mathrm{B}$ and exchange $\mathrm{J}$ interactions must be added. Collective properties may be obtained from magnetic susceptibilities $(x)$ measurements as a function of temperature $T$. Deviation from Curie-Law $(X \cdot T=C t e)$ indicate exchange interactions. At high temperature $(T>|\theta|)$ ferro and antiferromagnetic substances follow Curie-Weiss law $[X(T-\theta)=$ Ct $]$, their Weiss constant $\theta$ being respectively positive and negative (2)(3). However, a whole taxonomy of magnetism exists (3) (4). Let us mention metamagnets (3) (5), substances undergoing transition from an antiferromagnetic state to a ferromagnetic state when the applied magnetic field becomes larger than a critical value.

Organic stable free-radicals áre paramagnetic at hïgh temperature and follow Curie-Weiss Iaw (6) with negative Weiss constants. Positive Weiss constants are not common, although they are interesting because they indicate ferromagnetic interactions and perhaps ferromagnetism at low temperature. (For organic ferromagnets, see $(6,7)$ ). Few purely organic nitroxides are reported to have positive Weiss constants, and I would like to summarize what is known about their magnetic properties.

In 1967, with $\mathrm{H}$. Lemaire, we started an exploratory study of magnetic susceptibilities of nitroxide mono and biradicals, in collaboration with solid-state physicists. Similar studies on stable nitroxide had already been made on the first nitroxides as early as 1933 $(8-13)$. Negative Weiss constants were found $:-13(8), 0$ (9) or $-3 \pm 5$ (10) for dip-anisyl nitroxide 1 ; 0 (9) for dip-nitrophenyl nitroxide 2 ; 0 (10) or $-1,5$ (11) for Banfield and Kenyon radical $3 ;-12$ (12) or $-6 \pm 5$ (13) for porphírexide 4 and -90 (12)(13) for porphyrindine 5. Our measurements were made between $1,9 \mathrm{~K}$ and room temperature: Ketone 6 and carbonate biradical 7 followed Curie law. Tanol 8 and azine biradical 9 showed antiferromagnetic behaviour around $5 \mathrm{~K}$ and followed Curie-Weiss $1 \mathrm{aw}$, as well as suberate biradical 10 , their Weiss constant (reported as "Curie temperature" (14)) being $-6,-3$ and $+1 \mathrm{~K}$ respectively (14). Antiferromagnetic interactions in "Tano1" 8 were characterized (15) (see (16) and, for recent work (17)). Suberate's (10) positive Weiss constant suggested ferromagnetic interactions and possible ferromagnetism for $T<1{ }^{\circ} \mathrm{K}$. Further work was done by $\mathrm{C}$. Jeandey-Veyret during her thesis $(18-20)$. A magnetic transition was characterized at $\mathrm{T}=0.38 \pm 0,01 \mathrm{~K}(21)$ and attributed to ferromagnetic ordering. The corresponding ferromagnetic exchange interaction $0,18<J<0,35 \mathrm{~K}$ was determined. Further work in Grenoble showed that these ferromagnetic properties are fielddependent : below $0.35 \mathrm{~K}$. in zero field, 10 is antiferromagnetic and a transition occurs for fiels larger than ca $100 \mathrm{G}$. Its magnetic structure has been examined by neutron diffraction. In the crystal, molecule are arranged in sheets : within the sheets there are two ferromagnetic interactions between neighbours $\mathrm{J}_{1}=+1.1 \mathrm{~K}$ and $\mathrm{J}_{2}=0.07 \mathrm{~K}$ and between sheets, a sma11 antiferromagnetic interactions $\mathrm{J}^{\prime}=-0.015 \mathrm{~K}(22-24)$. Thus suberate 10 is the first purely organic metamagnet, with $2 \mathrm{D}$ ferromagnetic ordering.

Two other positive Weiss constant $\theta$ have been found by $C$. Jeandey-Veyret : Oxime 11 with $\theta=+0.45 \pm 0.05(18,19)$. shows a saturation curve (18) suggesting ferromagnetic interactions, but no further studies have been done. The copper-nitroxide complex 12 (25) has $\theta=+8.3$. Specific heat measurements for 12 have been interpreted in agreement with a ferromagnetic exchange interaction $(\mathrm{J}=+14.38 \overline{\mathrm{K}})$ between No and $\mathrm{Cu}$ spins and an antiferromagnetic one $(J=-0.02 \mathrm{~K})$ between No spins (18) (20). (Many other ferromagnetic interactions in nitroxide-transition metal complexes have been found by P. Rey and co-workers, e.g. (26)). A bis nitroxide 13 has $\theta=+0.5 \mathrm{~K}(27)$.

Very recently (27a) Awaga and Maruyama reported ferromagnetic interactions in 4-nitropheny1-nitronytnitroxide $13 \mathrm{a}$. A $\mu / \mathrm{T}$ curve (28) suggests ferromagnetic interactions in a nitroxide methacrylate monomer 14. I am not aware of other published positive Weiss constants $\theta$ and/or ferromagnetic interactions in solid-state nitroxides. Some organic radicals not containing transition elements have positive $\theta$ : galvinoxy (29), perchlorocarbon radicals 
(30), and a non d-metal compound, lithium phtalocyanine (31). (see also manganese phtaloxyarine (32)). In galvinoxy, ferromagnetic interactions have been detected (33). An organoselenium TCNQ is reported to show $2 \mathrm{D}$ ferromagnetism (34).

I now would like to report some recent work on bi and tetraradicals specially designed for their magnetic properties. In connexion with the search for organic ferromagnets, Miller, Epstein and Reiff (7) have recently pointed out the interest of radicals having $D_{2}$ symmetry. Some time ago (35), we reported such a nitroxide biradical 15. It had been designed because

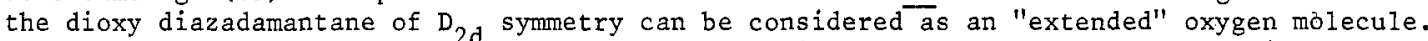
A triplet ground state was thus expected on the basis of degeneracy or orthogonality of the "magnetic orbitals" (35). Electron spin resonance (e.s.r.) measurements were interpreted as confirming this prediction and a ferromagnetic exchange interaction $2 \mathrm{~J}=170^{\circ} \mathrm{K}$ was deduced. However, intensity measurements by e.s.r. are not very precise and this study was complicated by the tendancy of unhidered, Bredt's rule-stabilized bicyclic nitroxides such as 16 (36) to dimerize in solution (37-41) or in solid state (37), (42-43), giving diamagnetic dimers (18), $(38-41)$, (44) with large (> 103 K), temperature-dependent singlet-triplet splitting (45-49). Bridge-head substituted bicyclic radicals such as 17 (38) do not dimerize appreciably in solution (38), (41), (44) but only in solid state (50), with a smaller singlet-triplet separation ( 200K) (45), (51).

We have thus prepared more hindered biradicals according to scheme (52). In dilute frozen solution, the new biradicals 18,19 and 20 display the expected triplet esr spectra, with the expected dipolar interactions ( $\overline{\mathrm{D} \sim} 230 \overline{\mathrm{G}})$. Esr signals are detected at the lowest available temperature. These biradicals are red paramagnetic solids at room temperature.

Magnetic properties of 18 have been investigated using a SQUID magnetometer (53). The results for pure 18 may be fitted to a Curie-Weiss law corresponding to 2 unpaired electrons per molecule and $\bar{\theta}=-5.5 \mathrm{~K}$. Although no ferromagnetic intramolecul interaction are detected in pure crystalline 18 , they cannot be excluded if antiferromagnetic intermolecular interactions become predominant at low temperature. With $\beta$ cyclodextrin, a complex containing 1 biradical and 2 cyclodextrins has been obtained, and intermolecular interactions are expected to be different. Here again the magnetic susceptibility $x$ follows a Curie-Weiss law, with a Curie constant $C=2.79$, slightly smaller than 3 expected for a radical-pair, and a positive Weiss constant $\theta=1.8 \mathrm{~K}$ indicating some ferromagnetic interaction in the complex. If these results are interpreted with an isolated radical-pair model, biradical 18 is a ground state triplet, with a singlet-triplet splitting of $7.2 \mathrm{~K}=4.7 \mathrm{~cm}^{-1}$. There may be other interpretations and further work is in progress in order to clarify this point. (Ferromagnetic intramolecular interactions have been repored in binitroxide 21 (54) and in Yang's biradical (55)).

Finally, I would like to report some nitroxide bi and tetraradical with characteristic dipolar interations. The strongest dipolar interaction $D=803 \mathrm{G}$ in nitroxide biradical 22 has been reported by J. Keana (56). A new biradical 23 , second with $\mathrm{D}=355 \mathrm{G}$. Other isomers 24,25 have $\mathrm{D}=167$ and $135 \mathrm{G}$ (57). (For a review of dipolar splitting in bisnitroxides, see (58)). Biradical $3 \mathrm{l}$ has almost $\mathrm{D}_{2 \mathrm{~h}}$ symetry $(71)$.

There are many nitraxide multiradicals (59-62) with more than two unpaired electrons, but to our knowledge, their No groups are too far apart for dipolar interactions to be detected,contrary to what is found in other multiradicals ((63-67) and references therein). Nitroxide tetraradicals with large dipolar interactions can easily be prepared from a diketone and a bisnitroxide precursor $26(68,69)$. Using diketone 27 , tetraradical 28 has been obtained (70). Its frozen solution spectrum extends on $735 \mathrm{G}$, 1arger than the total width (2D) of the corresponding biradicals $(29 \mathrm{D}=230 \mathrm{G}(68-69), 30 \mathrm{D}=121 \mathrm{G}(58))$. A splitting of $595 \mathrm{G}$ is measured, close to the sum of dipolar interactions (581G). Work is in progress to obtain dilute oriented samples in order to analyze the spectrum taking into account quintet, triplet and singlet states. The ground rate multiplicity has to be established.

Acknowledgements I thank my co-workers in Grenoble and Paris, whose name appear in the references.<smiles>COc1ccc(N([O-])c2ccc(OC)cc2)cc1</smiles>

1<smiles>CC1(C)C(=N)NC(=N)N1[O-]</smiles><smiles>O=[N+]([O-])c1ccc(N(O)c2ccc([N+](=O)[O-])cc2)cc1</smiles>

$\mathbf{e}$<smiles>CON1C(=NN=C2NC(=N)C(C)(C)N2[O-])NC(=N)C1(C)C</smiles>

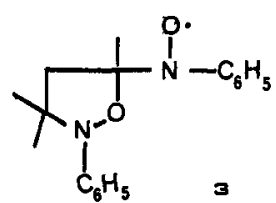<smiles>CC1(C)CC(=O)CC(C)(C)N1O</smiles> 
$\sum_{0-N_{0}^{-O}}^{\alpha}$

7<smiles>CC1(C)CC(O)CC(C)(C)N1[O]</smiles>

8

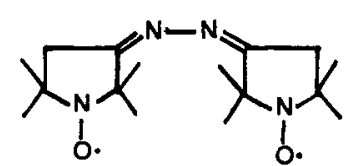

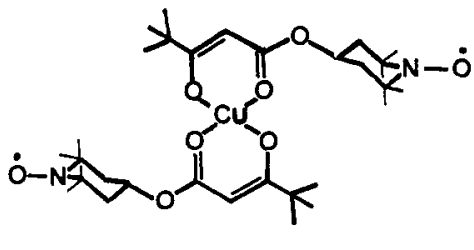

$1 \mathrm{e}$<smiles>C=C(C)C(=O)OC1CC(C)(C)N(O)C(C)(C)C1</smiles>

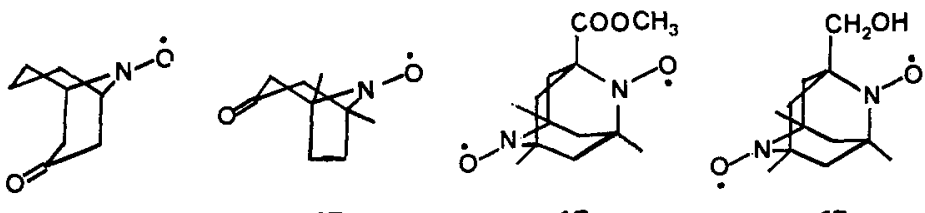
17<smiles>CC12CC3(C)CC(C)(CC(C)(C1)C(C)(C)N3[O])N2[O]</smiles>

20 21

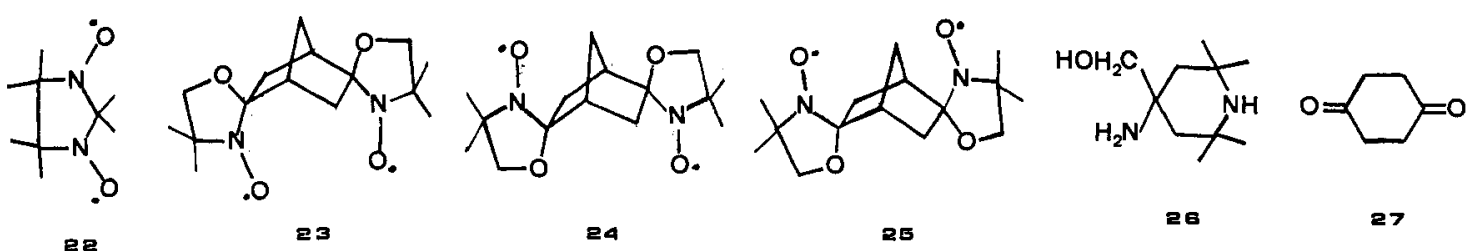

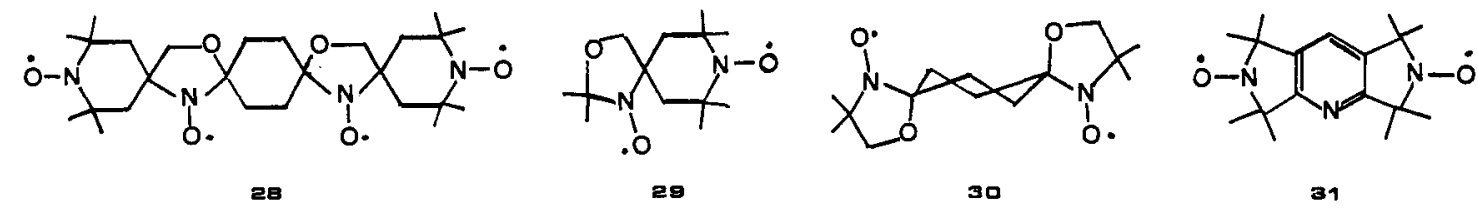

Scheme 1

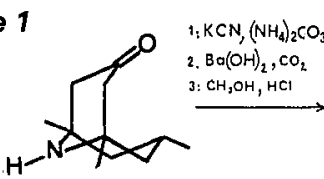<smiles>CCCC(N)CC(N)(CC(C)C)C(=O)OC</smiles>
$\mathrm{CH}_{2} \mathrm{OH}$

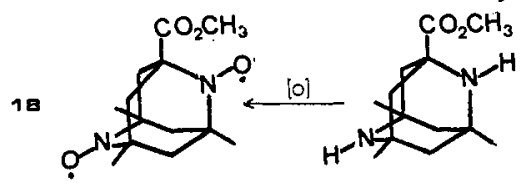

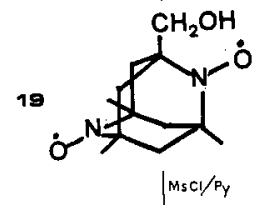<smiles>COCC12CC3(C)CC(C)(CC(C)(C3)N1O)N2O</smiles> 


\section{REFERENCES}

(1) H. Fischer, Landolt-Börstein Tab. (New Series), Group II, Volume 1, Springer-Verlag, Berlin, 1965, p.2†3.

(2) See for instance E. König, Landolt-Börstein Tab. (New Series), Group II, Volume 2, Springer-Verlag, Berlin, 1966, p, 173-1/12.

(3) Review : J.S. Miller, A.J. Epstein, W.M. Reiff, Chem. Rev., 88, 201 (1988).

(4) C.M. Hurd, in D.C. Mattis, The Theory of Magnetism II, Springer-Verlag, Berlin, 1985, cf. C.M. Hurd, Contemp. Phys. 23, 469 (1982).

(5) L. Néel, Nuovo Cimento 6N (Supp.), 942 (1957).

(6) A.L. Buchachenko, Khim. Fiz., 3, 3 (1984).

(7) Revaew : J.S. Miller, A.J. Epstein, W.M. Reiff, Accounts Chem. Res,, 21, 114 (1988).

(8) H. Katz, Z. Phys., 87, 238 (1934).

(9) L. Cambi, E. Tremolda, G. Devoto, Gazzetta Chim. Ital., 63, 579 (1933).

(10) E. Müller, I. Muiller-Rodloff, W. Bunge, Annalen, 520, $23 \overline{5}$ (1935).

(11) J. Van der Handel, Physica, 18, 921 (1952).

(12) E. Müller, I. Miiller-Rodlof f, Annalen, 521, 81 (1935).

(13) R. Kuhi, H. Katz, W. Franke, Naturwts. 22, 808 (1934).

(14) H. Lemaire, A. Rassat in A.B. Zahlan,Ed., The Triplet State, (Proc. Int. Symp. Amer. Univ. Beirtat, Feb. 14-19, 1967), Cambridge University Press, 1967, p.141.

(15) H. Lemaire, P. Rey, A. Rassat, A. de Combarieu, J.C. Michel, Mol. Phys, 14, 201 (1968).

(16) Y.S. Karimov, E.G. Rozantsev, Fiz. Tverd. Tela, 8, 2787 (1966), C.A. 66 6580.

(17) K. Takeda, N. Uryu, M. Inoue, Y. Yamauchi, J. Phys. Soc. Japan, 56, $7 \overline{36}$ (1987).

(18) C. Veyret, Thesis, Grenoble, $25 \mathrm{Feb} .1975, \frac{1}{\text { A.0. } 3359 .}$

(19) A. Blaise, H. Lemaire, J. Pilon, C. Veyret, Compt. Rendus Acad. Sci,, Paris, 274, 157 (1972).

(20) C. Veyret, A. Blaise, Mol. Phys., 25, 873-882 (1973). 1b is not a methyl ketone $\left(\mathrm{CO}-\mathrm{CH}_{3}\right)$ but the corresponding methyl ester $\left(\mathrm{CO}-0-\mathrm{CH}_{3}\right)$.

(21) M. Saint-Paul, C. Veyret, Phys. Letters 45A, 362 (1973). Due to a misprint in this paper's title, compound (32) is in fact $(22)$ in Buchachenko's review (6).

(22) C. Jeandey (née Veyret), J. Physique, 42, 875 (1981).

(23) G. Chouteau, C. Veyret-Jeandey, J. Physique, 42, 1441 (1981).

(24) A. Benoit, J. Flouquet, B. Gillon, J. Schweizer, J. Magn. Mag. Mater., 31-34, 1155. See also P.J. Brown, A. Capiomont, B. Gillon, J. Schweizer, Mol. Phys., 48, 753 (1983).

(25) R. Briere, A.M. Giroud, A. Rassat, P. Rey, Bull. Soc. Chim. Fr, 1980-II, 147. R. Briere A. Rassat, P. Rey, J. Am. Chem. Soc., 100, 343 (1978).

(26) e.g. A. Caneschi, D. Gatteschi, J.P. Renard, P. Rey, R. Sessoli, J. Am. Chem. Soc., 111, $785(1989)$.

(27) V.B. Strukoy S.Ya. Umansky, A.V. Zvorina, Chem. Phys. Letters, 18, 240 (1973).

(27a) K. Awaga, Y. Maruyama, to be published in J. Chem. Phys., 91 (15 August 1989). Gen. Phys. Adv. Abstracts 5 (13), 45 (1989).

(28) see fig.5, p.366 in M. Kamachi, M. Tamaki, Y. Morishima, S. Nozakura, W. Mori, M. Kishita, Polymer J., 14, 363 (1982).

(29) K. Mukai, Bu11. Chem. Soc. Japan, 42, 40 (1969).

(30) M. Ballester, J. Castäner, J. Riera, J. Veciana, Tetrahed. Lett., 1978, 479 M. Ballester, J. Castaner, J. Riera, A. Ibanez, Tetrahedron Lett. , $\frac{21}{21} 2435$ (1980) M. Ballester, J. Riera, J. Castaner, C. Onrubia, J.J. Ubierna, Tetrahedron Lett., 24, 2121 (1983)

M. Ballester, J. Veciana, J. Riera, J. Castaner, C. Rovira, O. Armet, J. Org. Chem., 51, 2472 (1986).

O. Armet, J. Veciana, C. Rovira, J. Riera, J. Castaner, E. Molins, J. Rius, C. Miravitilles, S. Olivella, J. Brichfeus, J. Phys. Chem., 91, 5608 (1987). see also L. Julia, M. Ballester, J. Castaner; J.L. Ortin, C. Onrubia, J. Org. Chem., 53, 1267 (1988).

(31) P. Turek, J.J. André, J. Simon, Solid State Comm., 63; 741 (1987)

P. Turek, M. Moussavi, J.J. André, Europhys. Lett., $, \frac{8}{4}, 275$ (1989).

(32) H. Miyoshi, Bul1. Chem. Soc. Japan, 47, 561 (1974).

(33) K. Awaga, T. Sugano, M. Kinoshita, J. Chem. Phys., 85, 2211 (1986), Chem. Phys. Letters $128,587(1986)$.

(34) S. Takagi, K. Kawabe, Phys. Letters, 59A, 70 (1976).

(35) R.M. Dupeyre, A. Rassat, J. Ronzaud, J. Amer.Chem. Soc., 96, 6559 (1974).

(36) R.M. Dupeyre, A. Rassat, J. Amer. Chem. Sac., 88, 3180 (1966).

(37) G.D. Mendeha11, K.U. Ingold, J. Amer. Chem. Soc., 95, 6390 (1973).

(38) A. Rassat, J. Ronzaud, Tetrahedron, 32, 239 (1976).

(39) R.M. Dupeyre, Thèse, Grenoble, 19 Dec. 1974, A.0. 10718.

(40) R.M. Dupeyre, A. Rassat, Tetrahedron, 34, 1501 (1978).

(41) R.M. Dupeyre, A. Rassat, Bul1. Soc. Chim. Fr., 1978-II, 612.

(42) A. Capiomont, B. Chion, J. Lajzerowicz, Acta Cryst., B27, 322 (1971).

(43) R.M. Dupeyre, A. Capiomont, J. Mol. Struct., 42, 243 (1977).

(44) A. Rassat, J. Ronzaud, J. Amer. Chem. Soc., 93, 5041 (1971)

(45) F. Genoud, Thèse, Grenoble (9 May 1980).

(46) F. Genoud, M. Decorps, Mol. Phys., 34, 1583 (1977).

(47) F. Genoud, Mo1. Phys., $\overline{43}, 1465(19 \overline{81})$. 
(48) A. Zvarina, V.B. Stryukov, D.N. Fedutin, A.B. Shapiro, JETP Lett., 19, 3 (1974).

(49) V.B. Stryukov, D.N. Fedutin, Fiz. tverd. Tela, 16, 2942 (1974).

(50) A. Capiomont, Acta Cryst., B29, 1720 (1973).

(51) J. Gloux, F. Genoud, M. Decorps, Mol. Phys., 42, 251 (1981).

(52) R. Chiarelii, A. Rassat, to be published.

(53) We thank Dr. P. Rey for access to his instrument.

(54) K. Mukai, H. Nagai, K. Ishizv, Butl. Chem. Soc. Japan, 48, 2381 (1975).

(55) K. Mukai, K. Ishizv, Y. Deguchi, J. Phys. Soc. Japan, 27,783 (1969).

(56) J.F.W. Keana, R.S. Norton, M. More110, D. Van Engen, J. Clardy, J. Amer. Chem. Soc., 100,934 (1978).

(57) G. Gagnaire, A. Rassat, to be published,

(58) J. Michon, P. Michon, A. Rassat, Nouv. J. Chimie, 4, 523 (1980).

(59) E.G. Rozantsev, "Free Nitroxyl Radicals", Plenum Press, New York, 1970.

(60) J.H. Van Raosmalen, E. Jones, H.J. Kevelam, Tetrahedron Lett., 19, 1865 (1972).

(61) V.D. Sholle, I.A. Prokop'ev, K.A. Sartova, S.S. Sarymsakov, E.G. Rosantzev, Izv. Akad. Nauk SSSR, Ser. Khim., 1981, 2578

(62) L. Dulog, H. Moller, Makromo1. Chem., 189, 2611 (1988).

(63) W. Wilker, G. Kothe, H. Zimmermann, Chem. Ber., 108, 2124 (1975).

(64) D.E. Seeger, P.M. Lahti, A.R. Rossi, J.A. Berson, J. Amer. Chem. Soc., 108, 1251 (1986).

(65) H. Murai, I. Safarik, M. Torres, 0.P. Strausz, J. Amer. Chem. Soc, 110, 1025 (1988).

(66) A. Izuoka, S. Murata, T. Sugawara, H. Iwamura, J. Amer. Chem. Soc., $\overline{109}, 2631$ (1987).

(67) Y. Teki, T. Takui, K. Itoh, H. Iwamura, K. Kobayashi, J. Amer. Chem. Soc., 108, 2147 (1986).

(68) J.F.W. Keana, R.J. Dinerstein, J. Amer. Chem. Soc., 93, 2808 (1971).

(69) J. Michon, A. Rassat, Tetrahedron, 36, 871 (1980).

(70) R. Chiarelli, A. Rassat, C. Rogier, to be published.

(71) C. Fabre, A. Rassat, to be published. 